\title{
EVALUATION FACTORS FOR MULTI-STAKEHOLDER BROADBAND VISUAL COMMUNICATION PROJECTS.
}

\author{
Marion A. Barfurth \\ University of Ottawa \\ Ottawa, Ontario. Canada \\ barfurth@uottawa.ca
}

\author{
Janice Singer, \\ Bruno Emond, \\ Norm Vinson, \\ Martin Brooks \\ National Research \\ Council Canada \\ Ottawa, Ontario. Canada \\ \{janice.singer, \\ bruno.emond, \\ norm.vinson, \\ martin.brooks\}@nrc.ca
}

\author{
John Spence \\ Communications \\ Research Center \\ Ottawa, Ontario. Canada \\ john.spence@crc.ca
}

\begin{abstract}
This paper presents a summary of multifaceted evaluation factors that we have identified through our research with Broadband Visual Communication (BVC) projects involving multiple stakeholders. The main benefit of these evaluation factors is that they provide a general evaluation framework for multiple stakeholder projects. The factors are social infrastructure, technical infrastructure, physical space, interaction style and content.
\end{abstract}

Keywords : Video
Communication,
Technologies, Field Study Evaluation, Multi-
stakeholder projects.

\section{INTRODUCTION}

Over the last two years the National Research Council of Canada's Institute of Information Technology (NRC-IIT), the Communications Research Centre and other partners have been involved in using and evaluating broadband video conferencing to support collaboration. While some authors (e.g., Kraut [4.8]) have focused on videomediated communication (VMC) and its relationship to face-to-face communication and co-location, our evaluative efforts have focused on field studies of VMC by authentic users solving real problems in a collaborative context.

Our research highlights five factors affecting the evaluation of VMC: social infrastructure, technical infrastructure, physical space, interaction style, and content. Each of these factors has been individually identified in the literature $[1,11$, 14]. However, we face the issue of characterizing and putting into operation all five simultaneously as each factor affects VMC to various degrees.

Another issue that arises in our evaluations is the diversity in success criteria introduced by multiple stakeholders. For example, technical support providers consider an event successful if there are no technical glitches, whether or not end-users of the technology successfully collaborate.

These two issues together, the five factors and the diversity in success criteria, pose the problem of creating and maintaining cohesion in an evaluation schema for multi-stakeholder Broadband Visual Communication (BVC) projects. What we mean by maintaining cohesion is that the multiple components present in an evaluation together reflect an accurate whole and yet each component also meets the evaluation needs of the individual stakeholders.

\subsection{Evaluation Backdrop}

The formal evaluations centered on two large multi-stakeholder projects. The first project was based on group-to-group collaboration in the context of professional development of teachers where teachers were geographically dispersed throughout the country. The second project focuses on point-to-point collaboration for the advanced teaching of music between a maestro violinist and his international students. Both 
projects have been evaluated via field studies [12, 14].

It is important to note that the events were structured around planned interactions, e.g., a formal lesson between a maestro and a student. The technology was not being used as a stand-in for co-location. Consequently, the goal was to promote collaborations without necessarily trying to replicate co-location. We are particularly interested in seeing how users adapt to technology to make collaboration effective. We expect our users to be motivated to make such adaptations because our users are authentic and have real needs to collaborate at a distance.

Because of the expense and limited availability of broadband VMC, our usage of the technology to date is focused on events. Events are collaborative activities scheduled at a precise time and for a specific duration. Synchronous collaborative activities involve people at two or more sites. This is made possible by connecting the sites with IPbased video conferencing.

In order to be successful, both social and technical aspects of events must be designed beforehand. Before the event occurs, the participants must determine schedules, and, in some cases, the activities themselves that will be supported by the technology (e.g. violin lesson). Metcalf and Morton [9] also talk about the importance of planning, recognizing its impact on an event.

Pre-event collaboration is also required to ensure that the technology functions appropriately. For example the various participating sites need to make sure that they are functional from a technological point of view. The factors that we identified earlier will influence both pre-event design and the actual collaborative event.

In the examples, we will be talking primarily about two groups of people. Implementers are the people who put together the broadband events. They include researchers, engineers, technical staff, administrative staff, and project leaders. Implementers are responsible for initiating, planning, designing and carrying out the technical aspects of the events. Implementers are also involved in the evaluation process. Users are the group of people who take part in the events. Their primary job is to be there and interact with others as well as to provide feedback for the evaluators.

An event will refer to one broadband session with the implementers implementing and tracking the technology and the users using the technology to collaborate on a task.

Evaluation was conducted via observation, instrumentation and userfeedback via surveys. We found that because of the multi-stakeholder nature of these projects, an evaluation plan was useful. The interests of each of the stakeholders were declared. Then, the evaluation plan identified parameters to put into operation each of these interests. For example, based on the different stakeholders' interests with respect to video connectivity, we identified several indicators corresponding to different sources of data; e.g., we measured whether the video transmission and reception were successful for all connected sites and whether the remote users could actually see each other. The first parameter was measured via monitoring equipment, the second via online surveys.

In the next section of the paper, we outline the five factors that we encountered with respect to effective evaluation for all stakeholders.

\section{FACTORS}

\subsection{Social Infrastructure}

For our events, the social infrastructure involves the social relationships between the implementers, the end-users and the stakeholders [11].

One critical aspect is motivation. End user has to perceive a benefit from using technology efficiently otherwise there's little participation. Motivation can come from interdependence of distributed members, political/organizational factors that would reward use of the technology and/or participation in a program. An example of motivation in our music project is that the maestro is too busy to travel and the students are highly motivated to learn from someone of this caliber. 


\subsection{Technical infrastructure}

The technical infrastructure includes the computer network architecture, hardware and software components. There could be a lot of variations among these components such as network bandwidth, compression algorithms, multicasting capability, communication protocols, type of hardware for supporting the communication (i.e., dedicated video-conferencing units, desktop computers), collaboration tools implemented in the software applications, etc.

In the context of the professional development project, the technology infrastructure used for professional development events included a CSCW application (Isabel) running over ca*net3, a pan Canadian network infrastructure. With our music project, tele-mentoring events used either video-conferencing units with additional sound mixing capabilities over ISDN network or computer applications with additional sound mixing capabilities over ca*net3.

Issues relating to quality of service are important to evaluate and cover a broad range of properties from a low level characterization of the network performance to evaluation of the user's experience. Both ends of the spectrum are related in an event setting and cannot really be treated separately.

\subsection{Physical Space}

Several factors that we have found important to evaluate for local space include the number of cameras and their position, the number of microphones and their quality, the size of the room, the lighting, and finally the type of furniture available. Evaluation of the virtual space looks at the quality of the 'telepresence' of the remote collaborators. Factors evaluated include the screen size, the size of the representation and its location on the screen and the position of the screen in the local room.

As an example of how physical space can affect collaboration, in our virtuoso training sessions, we found that the students and expert violinist needed to learn how to work in the physical space to actualize the lessons' potential. For instance, the maestro, already highly experienced with video conferencing, physically moves his mobile chair closer and further from the camera to create an effect that simulates what he would do if he were physically present to get the student's attention and focus. Although different than if the maestro were face-to-face, this technique has been developed to create a similar effect exploiting the possibilities of the physical space at hand.

\subsection{Interaction Style}

Interaction Style refers to two different aspects of event design. First, the implementers need to know how many users will be involved in the event at each of the multiple sites. For example, is the event a group-to-group event, a point-to-group event, an event that involves multiple sites or just two. This in turn defines the communication infrastructure.

The number of users involved will then, to a large degree, determine the format of the event. The format refers to the type of event envisioned. Will the event be a lecture, a meeting, a lesson, a training session, etc?

\subsection{Content}

Content refers to the content of the event. Will people be getting together to work on authentic problems, is there an immediate need for a solution to a problem, is the event focused on specific curriculum or a professional development need, etc. In most cases, authenticity of a task requiring some form of collaboration is at the core of the events.

Some content is more amenable to discussion, some to audio representation, some to visual representation, some to haptic representation, and some to a combination of the above modalities. This variation of content type serves two purposes. One is to meet the need of the participating users and the other is to push the development and capacities of the technical infrastructure. In our project with teachers, the content was the integration of project-based learning into classroom practice. 


\section{CONCLUSION}

Our experience with the factors affecting events and event design also suggests a research agenda. Additional research is required to better identify the factors that do have an effect, and to characterize and measure the strength of their effects. The factors are often difficult to evaluate as not all are always quantifiable and in some cases some are more amenable to description.

Past research has often used factorial experiments in evaluating these factors [6, $7,10,13]$. The consequence has been that the effects of one factor have been studied in isolation. In contrast, field studies, such as ours, require the simultaneous evaluation of all factors and the consideration that they may interact.

The factors are general enough to apply to video and other mediated or nonmediated interactions. The main difference with video-mediated communication is that the factors will have different weights. The physical settings of synchronous video communication for example will have a greater impact on the quality of experience than would other non video-mediated communication. Also Kraut's work [4, 8] demonstrates that media differences have an important impact on specific tasks involved in collaboration or other communication mediated activities.

In addition to the multiple-facets identified for effective evaluation of collaborative technology, evaluation tools that allow us to focus on the human to human collaborative process itself would potentially further our understanding of the processes inherent in collaborative enterprises [2,5]. This in turn will provide critical feedback for the design of tools and artifacts (virtual and other) that genuinely support both cognitive and social requirements of collaborative enterprises.

\section{References}

[1] J.S. Angiolillo, H.E. Blanchard, E.W. Israelski, A. Mane, "Technology Constraints of Video-Mediated Communication," in K.E. Finn, A.J. Sellen and S.B. Wilbur (Eds) Video-mediated Communication, Lawrence Erlbaum: NJ:Mahwah, 1997.
[2] M.A. Barfurth, "Understanding the collaborative learning process in a technology rich environment: The case of children's disagreements," in J.L. Schnase and E.L. Cunnius (Eds), Proceedings of the first international conference on Computer Support for Collaborative Learning (CSCL95), Hillsdale, NJ: Lawrence Erlbaum Associates, 1995, 8-13.

[3] K.E. Finn, "Introduction: An Overview of Video-Mediated Communication,", in K.E. Finn, A.J. Sellen and S.B. Wilbur (Eds) Video-mediated Communication, Lawrence Erlbaum: NJ:Mahwah, 1997.

[4] R. Fish, et al., "Video as a Technology for Informal Communication," Communications of the ACM, 36 (1), 1993

[5] J. Hollan, E. Hutchins, D. Kirsch, "Distributed Cognition: Toward a New Foundation for Human-Computer interaction Research." ACM Transactions on ComputerHuman Interaction, Vol 7, No 2, 2000.

[6] W. Huang, J. Olson, G. Olson, "Camera Angle Affects Dominance in Video-Mediated Communication," CHI '02, 2002.

[7]M. Jackson, A. Anderson, R. McEwan, J. Mullin, "Impact of Video Frame Rate on Communicative Behaviour in Two and Four Party Groups," CSCW '00, 2000.

[8] R. Kraut, et al., "Understanding Effects of Proximity on Collaboration: Implications for Technologies to Support Remote Collaborative Work," To appear in P. Hinds \& S. Kiesler (Eds.), Distributed Work, Cambridge MA: MIT Press.

[9]C.J. Metcalf, D.J. Morton, "Having Fun on the Access Grid: Building Informal

Relationships through the Beer Symposium," AG Retreat, 2002 [10]A. Monk, L. Watts, "A Poor Quality Video Link Affects Speech, But Not Gaze," CHI 95, 1995.

[11] G.M. Olson, J.S. Olson, "Making Sense of the Findings: Common Vocabulary Leads to the Synthesis Necessary for Theory Building," in K.E. Finn, A.J. Sellen and S.B. Wilbur (Eds) Video-mediated Communication, Lawrence Erlbaum: NJ:Mahwah, 1997.

[12] D. Pinelle, C. Gutwin, "A Review of Groupware Evaluations," WETICE Workshop on evaluating Collaborative Enterprises, 2000.

[13] R. Procter, et al. "An investigation of the Influence of Network Quality of Service on 
the Effectiveness of Multimedia

Communication," Group 99, 1999.

[14] A. Sellen, "Assessing Video-Mediated

Conduct: A discussion of different analytic approaches," in K.E. Finn, A.J. Sellen and S.B. Wilbur (Eds) Video-mediated Communication, Lawrence Erlbaum: NJ:Mahwah, 1997. 